\title{
Synthesis and characterization of functional copolymer/organo-silicate nanoarchitectures through interlamellar complex-radical (co)terpolymerization
}

\author{
E. Söylemez ${ }^{1}$, N. Çaylak ${ }^{2}$ Z. M. O. Rzayev ${ }^{*}$ \\ ${ }^{1}$ Department of Chemical Engineering, Faculty of Engineering, Hacettepe University, 06800 Ankara, Turkey \\ ${ }^{2}$ Department of Physics, Faculty of Art \& Science, Sakarya University, 54187 Sakarya, Turkey
}

Received 26 June 2008; accepted in revised form 27 July 2008

\begin{abstract}
The functional copolymers, having a combination of rigid/flexible linkages and an ability of complex-formation with interlayered surface of organo-silicate, and their nanocomposites have been synthesized by interlamellar complex-radical (co)terpolymerization of intercalated monomer complexes of maleic anhydride (MA) and itaconic acid (IA) with dimethyl dodecylamine surface modified montmorillonite (organo-MMT) (MA...DMDA-MMT and IA...DMDA-MMT) $n$-butyl methacrylate (BMA) and/or BMA/styrene monomer mixtures. The results of nanocomposite structure-composition-property relationship studies indicate that interlamellar complex-formation between anhydride/acid units and surface alkyl amine and rigid/flexible linkage balance in polymer chains are important factors providing the effective intercalation/exfoliation of the polymer chains into the silicate galleries, the formation of nanostructural hybrids with higher thermal stability, dynamic mechanical behaviour and well dispersed morphology.
\end{abstract}

Keywords: polymer synthesis, molecular engineering, interlamellar copolymerization, nanocomposites, thermal properties, dynamic mechanical properties

\section{Introduction}

In the last decade, the attention of many researchers focused on thermoplastic and thermoset polymer silicate layered (PLS), polymer/silica hybrid and polymer/carbon nanotube nanocomposites. This considerable scientific and engineering interest has been stimulated by possibility of the significant improvements in physical, mechanical, thermal and other important specific properties of PLS materials. The results of these researches were summarized and discussed in several reviews and articles [1-7]. PLSs are a class of organic-inorganic hybrids, composed of organic polymer matrix in which layered silicate particles of nano-scale dimension are embedded because these nanocomposites show enhanced mechanical properties [8,
9], gas barrier properties [10], and improved thermal stability [5, 11, 12], low flammability [11, 12] and protection effects from corrosion [13, 14].

Nowadays, organo-clays, especially organo-montmorillonite (MMT) are the most widely utilized silicates in polymer nanotechnology. MMT is a layered silicate crystal lattice which consists of two silica tetrahedral and one aluminium octahedral sheets with a plate-like structure of $1 \mathrm{~nm}$ thickness and $100 \mathrm{~nm}$ length [15]. The synthesis ways from functional monomers (maleic anhydride and its analogues) to polymer/clay (or silica) nanocomposites include three main methods such as (1) interlamellar (or intercalative, intragallery, etc.) copolymerization in the presence of various type of mineral silicates and organo-silicates, (2) intercalation 
of thermoplastic polymers in melt by reactive extrusion in situ processing and (3) sol(hydro)-gel method, i.e., reactions of anhydride (or carboxylic group)-containing polymers with organosilanes (polymer/silica hybrid synthesis). Last two methods, especially intercalation of polymers, are the most promising ways to synthesize polymer/intercalant-clay nanocomposites on the base of graft copolymers of maleic anhydride and its analogues as reactive compatibilizers.

Although the most promising reaction to great polymer/clay nanocomposites is the in situ polymerization of functional monomers, such as styrene [16, 17], $\mathrm{N}$-vinylcarbazole [18], 4-vinyl-pyridine [19], methyl methacrylate [20, 21], acrylonitrile [22] and $N$ - $n$-butylmaleimide [23], as well as synthesis of low-molecular-weight polyamide 6/hydrotalcite intercalated nanocomposites via in situ polymerization [24], whereas the intercalative radical copolymerization of binary or ternary monomer systems in the presence of mineral clay has been scarcely investigated. The polymerization of acrylate [25, 26], styrene-4-sulfonate [27], sulfopropyl methacrylate [28] and acrylic acid [29] in the inorganic layered materials have also been investigated as organic/inorganic nanocomposite materials. $\mathrm{Yu}$ et al. [29] completely polymerized the acrylic acid regularly fixed in layered aminopropylsilica without initiator at $100^{\circ} \mathrm{C}$ for $24 \mathrm{~h}$ to form a novel organic/inorganic nanocomposite named as layered polyacrylamide propylsilica. Examples for interlamellar polymerization of the binary monomer systems are bulk copolymerization of styrenemethyl methacrylate $[23,27,30]$, emulsion copolymerization of styrene-acrylonitrile [31], styrene- phenyl maleimide (PhMI) [32] and styrene-butyl methacrylate [33] monomer pairs. It was observed that the polymerization rate was accelerated by the addition of the clay in reaction medium; this addition also significantly influenced the structure and properties of the prepared polymer nanocomposites [23, 27, 34]. Liu et al. [35] investigated the bulk, solution and emulsion copolymerization of styrene with methyl methacrylate and $\mathrm{N}$-phenylmaleimide (PhMI) (at 1:9 molar PhMI/styrene feed ratio) in the presence of organo(cetyltrimethyl ammonium bromide)-modified MMT or $\mathrm{Na}^{+}$-MMT in order to prepare nanocomposites with good dispersability of clay. According to the authors, the catalytic effect of the clay on a polymerization may be mostly caused by the small-size effect of the clay layers, and the active sites are on the surface of the clay particles rather than between layers. Wang et al. $[36,37]$ showed that a comparison of solution, emulsion, suspension and bulk polymerization along the nanocomposites may also be prepared by melt in situ reactive blending of monomer/polymer/clay mixtures.

Recently, we have reported the synthesis of the functional copolymer/silica (or organo-silicate) hybrids and polypropylene/organo-MMT nanocomposites by (1) reaction of anhydride-containing copolymers with $\gamma$-aminopropyltriethoxysilane [38-40], (2) interlamellar copolymerization of maleic anhydride with some vinyl and acrylic comonomers [40] and (3) reactive extrusion in situ processing $[4,40]$. In this work, we present (a) the synthesis of poly[maleic anhydride (MA)-co-nbutylmethacrylate (BMA)/organo-MMT, poly(MAalt-styrene-co-BMA)/organo-MMT, and poly[ita-

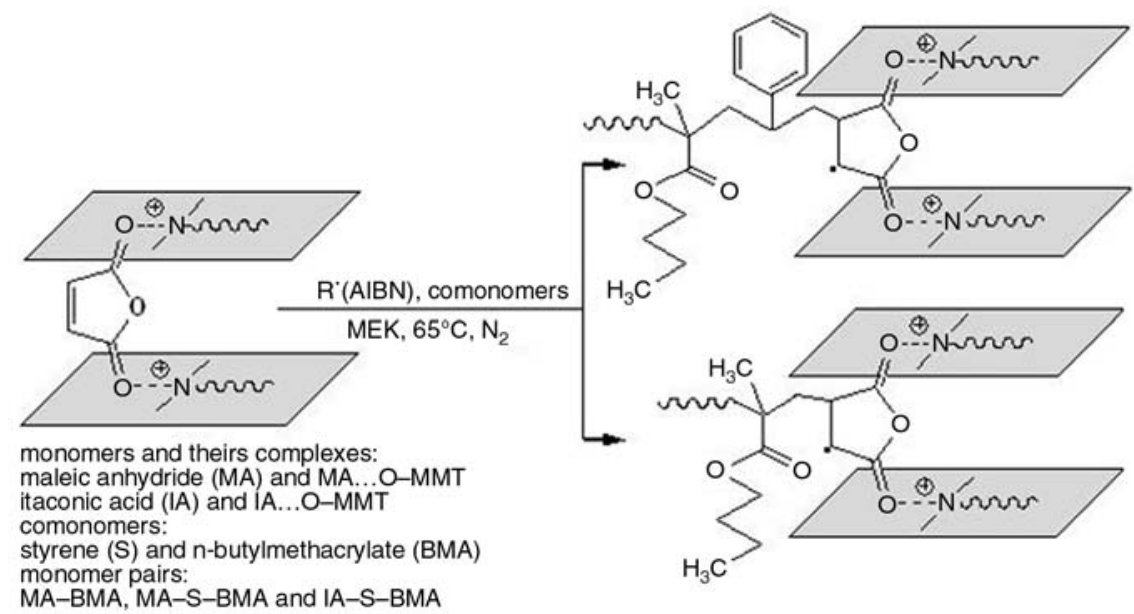

Figure 1. Schematic representation of interlamellar complex-radical (co)terpolymerizations 
conic acid (IA)-co-styrene-co-BMA)]/organoMMT nanoarchitectures prepared by complex-radical interlamellar copolymerization, (b) the results of the copolymer composition-nanostructure formation relationship studies, and (c) characterization of (co)terpolymer/organo-MMT nanocomposites by thermal (DSC and TGA-DTG), dynamic mechanical (DMA), X-ray diffraction (XRD) and scanning electron microscopy (SEM) analysis methods. Special attention is paid to the role of complex formation of MA (or IA) and its (co)terpolymers with dimethyldodecylamine (DMDA) surface modified silicate layers in interlamellar co(ter)polymerization reactions and intercalation/ exfoliation of polymer chains between silicate galleries. Taking into consideration of this effect, general scheme of interlamellar (co)terpolymerizations can be represented as shown in Figure 1.

\section{Experimental}

\subsection{Materials}

$\mathrm{S}$ and BMA monomers (Aldrich) were purified before use by distillation under moderate vacuum. MA and IA monomers were obtained from Fluka (Switzerland). MA was purified by recrystallization from anhydrous benzene and sublimation in vacuum. IA monomer was purified by recrystallization from distilled water solution and drying at $60^{\circ} \mathrm{C}$. The $\alpha, \alpha^{\prime}$-azoisobutyronitrile (AIBN) (Fluka) was recrystallized twice before use from methanol. DMDA-MMT (Viscobent SB-1) was purchased from Bensan (Enez, Turkey) having the following average parameters: specific surface area $43.6 \mathrm{~m}^{2} \cdot \mathrm{g}^{-1}$, specific mesopore volume $0.14 \mathrm{~cm}^{3} \cdot \mathrm{g}^{-1}$, content of $\mathrm{N} 1.12 \%$ and $\mathrm{C} 32.56 \%$, crystallinity $58.2 \%$ (by XRD), melting point $\left(T_{m}\right)$ $160^{\circ} \mathrm{C}$ (by DSC for the surface alkyl ammonium complex), temperature of decomposition for $T_{d(\text { onset })} 238^{\circ} \mathrm{C}$ and $T_{d(\max )} 361^{\circ} \mathrm{C}$ (by TGA). All other solvents and reagents were of analytical grade and used without purification.

\subsection{Terpolymerization procedure}

Terpolymerization of MA, S and BMA using given molar monomer feed ratios of (MA (or IA):S): BMA $=(1: 1): 1.23-5.0$ were carried out in methyl ethyl ketone (MEK) or in $N, N^{\prime}$-dimethylformamide (DMF) (for IA/basic monomer system) with AIBN as an initiator at constant total concentration of monomers under nitrogen atmosphere. Reaction conditions: $[M]_{\text {total }}=2.86-3.04 \mathrm{~mol} \cdot \mathrm{l}^{-1},[\mathrm{AIBN}]=$ $2.03 \cdot 10^{-2} \mathrm{~mol} \cdot \mathrm{l}^{-1}, \quad[$ Solvent $] /[M]=3, \quad$ molar monomer ratios of $(\mathrm{MA}: \mathrm{S}) / \mathrm{BMA}=0.20-0.81$, reaction time $24 \mathrm{~h}$, and conversion around 75-92 wt\%. Appropriate quantities of monomers, solvent (MEK or DMF) and AIBN were placed in a standard Pyrex-glass reactor, and was cooled by liquid nitrogen and flushed with dried nitrogen gas for at least $3 \mathrm{~min}$, then soldered and placed in a carousel type microreactor with a thermostated heater and magnetic mixer. The reaction mixture was stirred at $65 \pm 0.1^{\circ} \mathrm{C}$ for $24 \mathrm{~h}$. The resulting terpolymers were isolated from reaction mixture by precipitating with diethyl ether, then washed with several portions of benzene and dried at $40^{\circ} \mathrm{C}$ under vacuum. The terpolymer compositions were found by chemical (acid number for MA units) analysis, and ${ }^{1} \mathrm{H}$ NMR spectroscopy (Figure 2) using integral area of chemical shifts of monomer functional groups for quantitative analysis. Under similar conditions, poly(MA-co-BMA) with given composi-
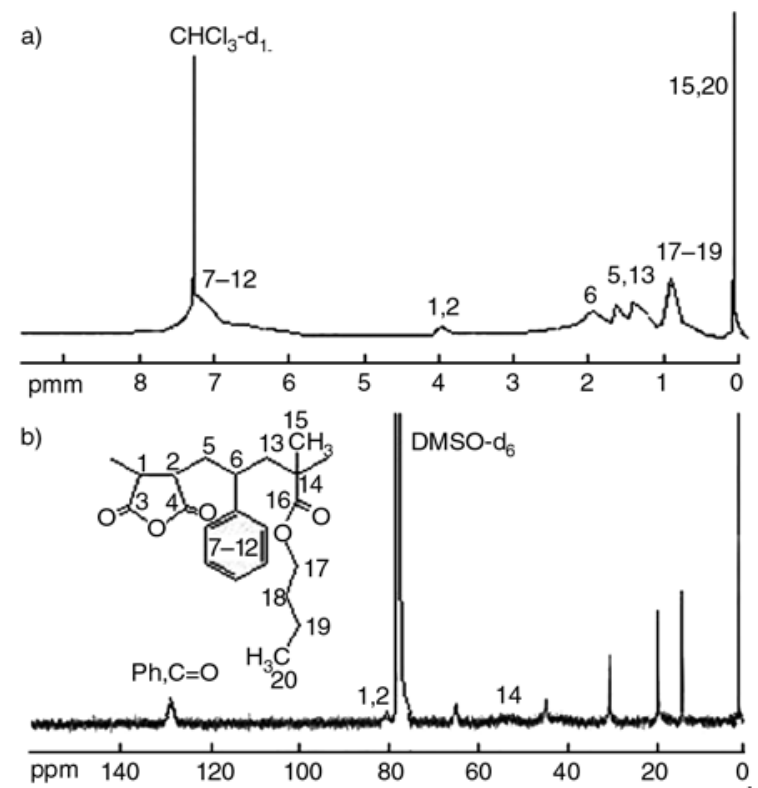

c)

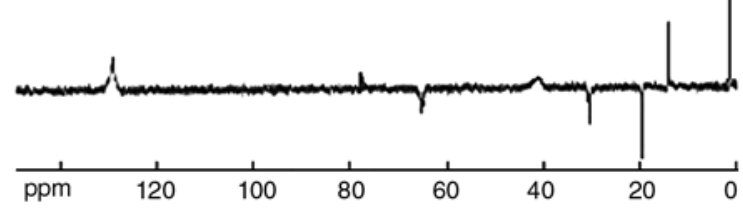

Figure 2. (a) ${ }^{1} \mathrm{H} \mathrm{NMR}$ (in $\mathrm{CHCl}_{3}-d_{1}$ ), (b) ${ }^{13} \mathrm{C} \mathrm{NMR}$ (in DMSO- $d_{6}$ ) and (c) ${ }^{13} \mathrm{C}$ NMR-DEPT-135 (in DMSO- $d_{6}, \mathrm{CH}_{2}$ region in negative position) spectra of poly(MA-alt-S-co-BMA) 
Table 1. Some characteristics of the binary and ternary copolymers

\begin{tabular}{|c|c|c|c|c|}
\hline (Co)terpolymers & $\begin{array}{l}\text { BMA-unit [mol\%] } \\
\text { (unit ratio) }\end{array}$ & 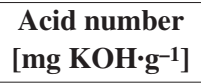 & $\begin{array}{c}{[\eta]_{\text {in }}\left[\mathrm{g} \cdot \mathrm{dl}^{-1}\right]} \\
\text { in DMF at } 25 \pm 0.1^{\circ} \mathrm{C}\end{array}$ & $\begin{array}{c}\mathbf{T}_{\mathrm{g}}\left[{ }^{\circ} \mathbf{C}\right] \\
(\text { by DSC) }\end{array}$ \\
\hline Poly(MA-co-BMA) & $\begin{array}{c}38.5 \\
(1: 1.25)\end{array}$ & 398 & 0.235 & 59.0 \\
\hline Poly(MA-alt-S-co-BMA)-1 & $\begin{array}{c}36.7 \\
(1: 1: 1.16)\end{array}$ & 301 & 0.384 & 122.5 \\
\hline Poly(MA-alt-S-co-BMA)-2 & $\begin{array}{c}54.2 \\
(1: 1: 2.37) \\
\end{array}$ & 205 & 0.352 & 100.6 \\
\hline Poly(MA-alt-S-co-BMA)-3 & $\begin{array}{c}71.1 \\
(1: 1: 4.92)\end{array}$ & 125 & 0.305 & 80.2 \\
\hline
\end{tabular}

tion was synthesized. Some characteristics of synthesized (co)terpolymers are presented in Table 1.

\subsection{Interlamellar (co)terpolymerization procedure}

MA (or IA) monomer was dispersed in MEK (or DMF)/DMDA-MMT mixture at monomer/alkyl amine molar ratio of $1: 2$ by intensive mixing at $40^{\circ} \mathrm{C}$ for $3 \mathrm{~h}$ before reaction to prepare the intercalated complex of monomer with surface alkyl amine. Then appropriate amount of S and BMA comonomers and AIBN (1.0 wt \%) was added to reaction mixture containing organo-MMT...MA (or IA) complex and heated with mixing at $65^{\circ} \mathrm{C}$ under nitrogen atmosphere for $24 \mathrm{~h}$. Prepared composition was isolated by precipitation with diethyl ether, then washed with several portions of benzene and dried under vacuum at $60^{\circ} \mathrm{C}$.

\subsection{Characterization}

FT-IR spectra of the (co)terpolymers ( $\mathrm{KBr}$ pellet) were recorded with FT-IR Nicolet 510 spectrometer in the $4000-400 \mathrm{~cm}^{-1}$ range, where 30 scans were taken at $4 \mathrm{~cm}^{-1}$ resolution. ${ }^{1} \mathrm{H}\left\{{ }^{13} \mathrm{C}\right.$ and ${ }^{13} \mathrm{C}$ DEPT-135 $\}$ NMR spectra were performed on a JEOL 6X-400 (400 MHz) spectrometer with $\mathrm{CHCl}_{3}-d_{1}$ or DMSO- $d_{6}$ as a solvent at $25^{\circ} \mathrm{C}$. Molar fractions of the comonomer units $\left[m_{1}\right.$ and $m_{2}$, where $m_{1}$ is (MA-S) alternating diad and $m_{2}$ is BMA unit] in terpolymers were calculated according to the Equation (1) using NMR analysis data:

$$
\frac{m_{1}}{m_{2}}=\frac{n_{2} A m_{1}(\mathrm{Ph} \text { or } \mathrm{CH})}{n_{1} A m_{2}\left(\mathrm{CH}_{3}\right)}
$$

where $n_{1}$ and $n_{2}$ are the integers of proton(s) in the functional groups of the monomer units, $A_{m 1}$ and $A_{m 2}$ are the integral areas per proton from corresponding groups of the monomer unit regions in ${ }^{1} \mathrm{H}$ NMR spectra.

Acid numbers of the anhydride (or carboxylic)containing (co)terpolymers were determined by standard titration method. Intrinsic viscosities of the (co)terpolymers with different compositions were determined in DMF at $25 \pm 0.1^{\circ} \mathrm{C}$ within the concentration range of $0.05-1.5 \mathrm{~g} \cdot \mathrm{dl}^{-1}$ using an Ubbelohde viscometer. Thermogravimetric (TGA) and differential thermogravimetric (DTG) analyses were performed on a Setaram Labsys TG-DTA 12 Thermal Analyzser (France) at a heating rate of $5^{\circ} \mathrm{C} / \mathrm{min}$, under nitrogen atmosphere. The differential scanning calorimetry (DSC) was performed on a Shimadzu calorimeter (Japan). Dynamic mechanical analysis (DMA) was performed on a TA Instrument-Q800. Scanning electron microscopy (SEM) patterns were recorded using a JSM-6400 JEOL SEM with an acceleration voltage of $30 \mathrm{kV}$.

The X-ray diffraction (XRD) patterns were obtained from a Rigaku D-Max 2200 powder diffractometer. The XRD diffractograms were measured at $2 \theta$, in the range $1-50^{\circ}$, using a $\mathrm{CuK}_{\alpha}$ incident beam $(\lambda=1.5406 \AA)$, monochromated by a Ni-filter. The scanning speed was $1 \%$ min, and the voltage and current of the X-ray tubes were $40 \mathrm{kV}$ and $30 \mathrm{~mA}$, respectively. The Bragg equation was used to calculate the interlayer spacing $(d): n \lambda=$ $2 d \sin \theta$, where $n$ is the order of reflection, and $\theta$ is the angle of reflection. Crystallinity of the nanocomposites was calculated using the Equations (2) and (3) [41]: 


$$
\chi_{c}=\frac{\int_{0}^{\infty} s^{2} I_{c}(s) d}{\int_{0}^{\infty} s^{2} I(s) d}
$$

where $s$ is the magnitude of the reciprocal-lattice vector which is given by $s=(2 \sin \theta) / \lambda(\theta$ is one-half the angle of deviation of the diffracted rays from the incident $\mathrm{X}$-rays and $\lambda$ is the wavelength); $I(s)$ and $I_{c}(s)$ are the intensities of coherent X-ray scattering from both crystalline and amorphous regions and from only crystalline region of polymer sample, respectively, and $d$ is interlayer spacing.

$\chi_{c}=\frac{W_{c}}{W_{c}+W_{a}} \cdot 100$

where $W_{c}$ and $W_{a}$ are the areas of the crystalline and amorphous portions in the X-ray diffractogram, respectively.

Intercalation or exfoliation degree (ED) was calculated according to the Equation (4):

$\mathrm{ED}[\%]=\frac{I_{e}}{I_{e}+I_{0}} \cdot 10^{-2}$

where $I_{e}$ (or $I_{c}$ ) and $I_{0}$ are the intensity of the diffraction peaks associated with the exfoliated and non-exfoliated structures at corresponding $2 \theta$ values.

\section{Results and discussion}

\subsection{Complex-radical interlamellar copolymerization}

Intercalation and exfoliation of functional polymer chains between surface modified silicate layers are one of the most important processes proceeding through chemical or physical interactions of functional groups from the monomers and/or growing polymer chains with surface alkyl amine in the interlamellar copolymerization. Character of this interaction strongly depends on the nature and functionality of the chosen monomer and polymer chains, which may provide an effective interaction with silicate layers via complex-formation, hydrogen bonding, and amidization/imidization reactions (in the case of primary alkyl amine modified MMT
[4]). Effect of H-bonding in ternary solvent/protondonor polymer/proton-acceptor polymer system was also reported by Figueruelo et al. [42]. Taking this principle into consideration, we have investigated the radical-initiated interlamellar copolymerization of MA as a strong hydrophilic electronacceptor monomer with BMA as an amphiphilic comonomer and interlamellar copolymerization of two monomer systems such as MA-BMA and itaconic acid (IA as a hydrophilic electron-acceptor monomer capable of H-bonding)-BMA in the presence of DMDA surface modified MMT silicate layers as a surfactant with positive charge tertiary amine group ending. The functional copolymers, having a combination of rigid/flexible linkages and an ability of complex-formation with interlayered surface of organo-silicate, and their nanocomposites have been synthesized by interlamellar complex-radical copolymerization of MA or IA (acceptor monomer) and $n$-butyl methacrylate (BMAacceptor) in MEK (or DMF) at $70^{\circ} \mathrm{C}$ in the interlayered region of DMDA surface modified MMT (DMDA-MMT) used as a nano-reactor (Figure 1). To elucidate the effect of DMDA-MMT in copolymerization and its interaction with functional monomers, especially with MA and IA monomer units, and in the formation of nano-structures, we also synthesized pure copolymers under similar conditions without DMDA-MMT for the comparative analysis of the obtained results. It was observed that copolymerization rate and copolymer conversion significantly depend on the presence of DMDA-MMT. Addition of DMDA-MMT as a complex-forming agent essentially increases the copolymer yield. This fact can be explained by the catalytic effect of organo-MMT as a complexforming agent, which is similar to the well known effect of Lewis acids in complex-radical polymerizations [43]. Catalytic effect of organo-silicates in radical polymerization of vinyl and acrylic monomers have also been observed by many researchers [23, 27, 34, 35].

In order to increase the effectivity of intercalation process and therefore, the formation of nano-structures in the studied system, MA and IA monomers were dispersed between silicate galleries before copolymerization reactions to prepare the fixed interlayer anhydride/alkyl amine (MA...DMDAMMT) and carboxylic acid/alkyl amine (IA... DMDA-MMT through strong H-bonding) com- 


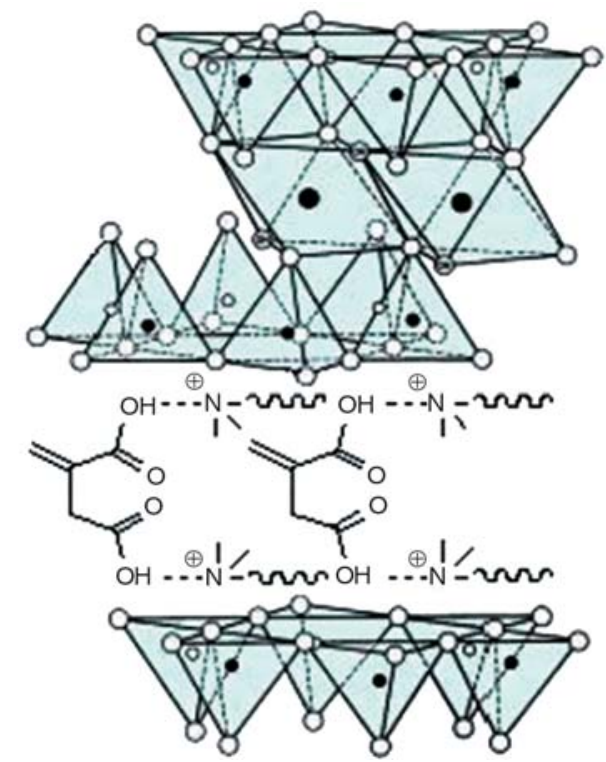

Figure 3. Interlamellar complex-formation via H-bonding in the IA/DMDA-MMT system

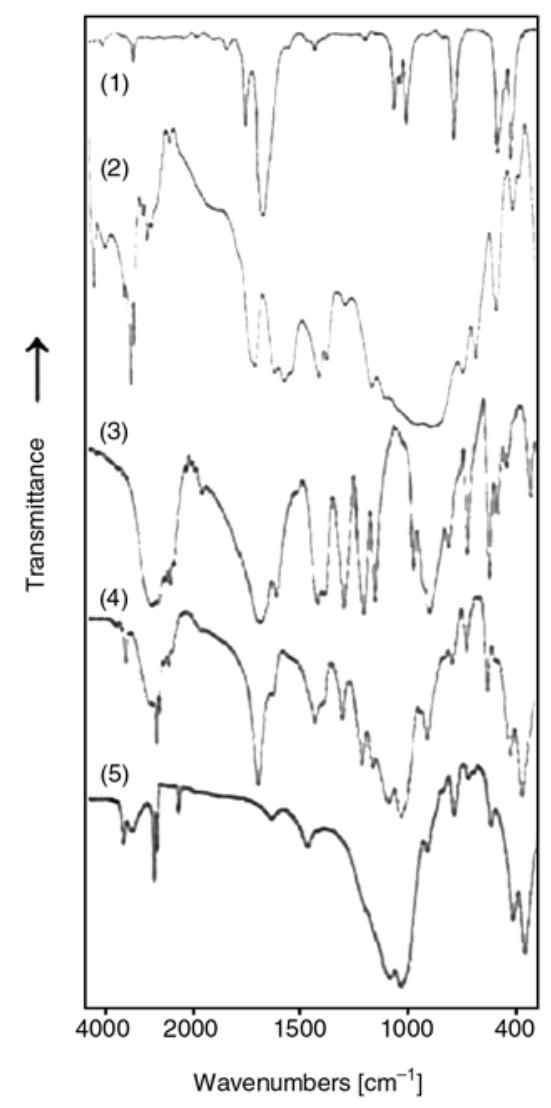

Figure 4. FTIR spectra of (1) MA monomer,

(2) MA...DMDA-MMT complex, (3) IA monomer, (4) IA...DMDA-MMT complex and (5) pristine DMDA-MMT; effect of interlamellar complex-formation

plexes (Figure 3; here, well known lattice structural model of Hendricks for MMT was used [44]).
In this case, two carbonyl or carboxylic groups of the MA (or IA) monomer molecule interact with two neighbouring silicate platelet surfaces having tetrahedral structures, which contain alkyl ammonium cations, through interlayer complex or strong H-bonding. Structure of these molecular complexes was confirmed by FTIR spectroscopy (Figure 4). From comparative analysis of the spectra of free IA monomer, virgin DMDA-MMT and IA/DMDAMMT system, the following changes of the characteristic bands were observed: (1) due to the formation of the interlayer molecular complexes, very broad $1705 \mathrm{~cm}^{-1}$ band $(\mathrm{C}=\mathrm{O}$ in $-\mathrm{COOH})$ of free IA monomer appeared in the spectra of IA-DMDAMMT system in the form of a strong narrow band; (2) characteristic $\mathrm{CH}_{2}=\mathrm{C}<$ band at $1630 \mathrm{~cm}^{-1}$ for IA monomer shifted to $1636 \mathrm{~cm}^{-1}$ region due to the change of the conjugation effect of double bond with carbonyl group after its complex-formation with surface alkyl amine cations; (3) broad band at $3475 \mathrm{~cm}^{-1}$ associated with $\mathrm{OH}$ groups of DMDAMMT disappeared, and a new broad band formed at $3125 \mathrm{~cm}^{-1}$, overlapping with two weak bands for stretching ammonium cation and $\mathrm{CH}_{2}=$ bands, respectively; (4) appearance of broad band at $4125 \mathrm{~cm}^{-1}$ can be related to complexed $\mathrm{OH}$ band in $-\mathrm{COOH}$ groups; (5) appearance of new bands at 1565-1470 (w) and $1400 \mathrm{~cm}^{-1}(\mathrm{~m})$ related to the $\mathrm{C}-\mathrm{N}$ deformation and $-\mathrm{COO}-$ sym. stretching bands, respectively, in $-\left(\mathrm{CH}_{2}\right)_{\mathrm{n}}-\mathrm{N}^{+} \cdot-\mathrm{OOC}-$ complex.

Similar effect is obtained from comparative analysis of FT-IR spectra of the (co)terpolymers and their nanocompsities which are illustrated in Figure 5. The FT-IR spectra of poly(MA-alt-S-coBMA), DMDA-MMT and their hybrid nanocomposite indicated the following changes of characteristic bands: (1) very broad absorption band around $1250-1000 \mathrm{~cm}^{-1}$ observed in the $\mathrm{Si}-\mathrm{O}$ stretching region for virgin DMDA-MMT is transferred to relatively narrow doublet peak after interlamellar copolymerization, (2) appearance of a new band at $1625 \mathrm{~cm}^{-1}$ which is associated with complexed $\mathrm{C}=\mathrm{O}$ band in $-\mathrm{C}=\mathrm{O} \ldots \mathrm{N}^{+}-$complex as a result of in situ interaction between polar anhydride (or ester) unit and alkyl ammonium linkage, (3) presence of 850 and $800 \mathrm{~cm}^{-1}$ bands due to in situ esterification reaction of anhydride units of intercalated terpolymer macromolecules with surface silicate $\mathrm{Si}-\mathrm{OH}$ groups, and (4) disappearance of $1633 \mathrm{~cm}^{-1}$ band 


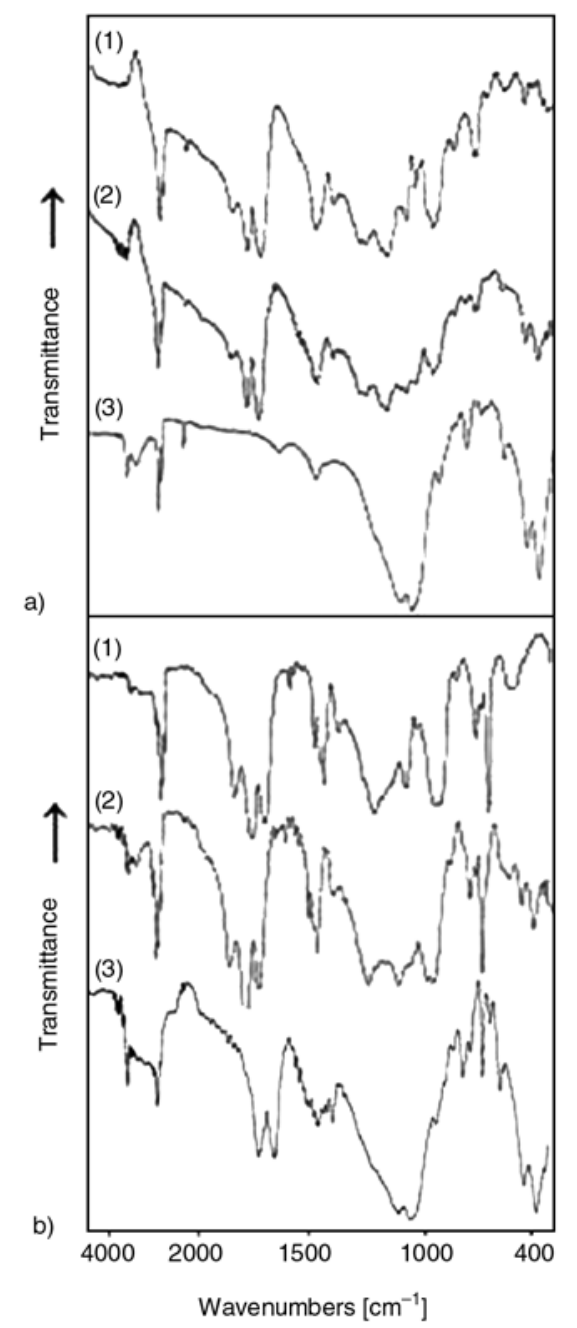

Figure 5. FTIR spectra of (a): (1) copolymer-1, (2) terpolymer-1, (3) virgin DMDA-MMT and (b): (1) copolymer-1/DMDA-MMT, (2) terpolymer1/DMDA-MMT, (3) terpolymer-IA-1/DMDAMMT (water OH group) in DMDA-MMT spectra after intercalation/exfoliation due to the possible hydrolysis of anhydride units. These observed changes can serve as a reasonable agreement confirming the delamination of silicate layers due to the intercalation of the polar terpolymer macromolecules through in situ complexing with positive charged tertiary amine groups. On the other hand, hydrolysis and esterification of anhydride units with interlayer water molecules and $\mathrm{Si}-\mathrm{OH}$ groups, respectively, in the surface of silicate layers during complex-radical interlamellar terpolymerization in situ processing are also possible.

\subsection{Nanocomposite structure-thermal behaviour relationship}

Studied (co)terpolymers consisting of an alternate sequence of rigid (MA or IA units and phenyl rings) and flexible ( $n$-butylester side-chain and backbound main chain) molecular linkages can exhibit a liquid-crystalline behaviour [45]. Results of DSC analysis of virgin (co)terpolymers and their nanocomposites, illustrated in Figure 6 and summarized in Table 2, can serve as a reasonable agreement for this proposal. In both poly(MA-co-BMA) (C-1) and its nanocomposite (C-1-NC) DSC curves, the characteristic peaks at 59.0 and $63.0^{\circ} \mathrm{C}$ appeared, which can be related to glass transition $\left(T_{g}\right)$ and melting point $\left(T_{m}\right)$ temperatures for the amorphous and semi-crystalline copolymer and its nanocomposite, respectively. However, both systems did not exhibit recrystallization behaviour after reverse cooling of polymer samples from

Table 2. Thermal behaviour of the (co)terpolymers and their nanocomposites with constant amount of the DMDA-MMT (5.0 wt \%)

\begin{tabular}{|c|c|c|c|c|c|c|c|}
\hline \multirow{3}{*}{ Nanocomposite } & \multicolumn{2}{|c|}{ DSC analysis } & \multicolumn{5}{|c|}{ TGA analysis } \\
\hline & \multirow{2}{*}{$\begin{array}{c}\mathbf{T}_{\mathbf{m}} \\
{\left[{ }^{\circ} \mathbf{C}\right]}\end{array}$} & \multirow{2}{*}{$\begin{array}{c}\Delta \mathbf{H} \\
{[\mathbf{J} / \mathbf{g}]}\end{array}$} & \multirow{2}{*}{$\begin{array}{c}\mathbf{T}_{\mathbf{d} \text { (onset) }} \\
{\left[{ }^{\circ} \mathbf{C}\right]}\end{array}$} & \multirow{2}{*}{$\begin{array}{c}\mathbf{T}_{\mathbf{d}(\max )} \\
{\left[{ }^{\circ} \mathbf{C}\right]}\end{array}$} & \multicolumn{3}{|c|}{ Weight loss $[\%]$ at $\left[{ }^{\circ} \mathrm{C}\right]$} \\
\hline & & & & & 250 & 300 & 350 \\
\hline Poly(MA-co-BMA)/organo-MMT & $63.0^{\mathrm{a}}$ & - & $\begin{array}{c}271 \\
(262)^{\mathrm{b}}\end{array}$ & $\begin{array}{c}381 \\
(362)\end{array}$ & $\begin{array}{c}0.0 \\
(0.0)\end{array}$ & $\begin{array}{c}3.2 \\
(4.4)\end{array}$ & $\begin{array}{c}23.8 \\
(36.2)\end{array}$ \\
\hline Poly(MA-alt-S-co-BMA)-1/organo-MMT & 143.2 & 10.9 & $\begin{array}{c}274 \\
(214)\end{array}$ & $\begin{array}{c}427 \\
(373)\end{array}$ & $\begin{array}{c}0.0 \\
(2.1)\end{array}$ & $\begin{array}{c}5.9 \\
(7.3)\end{array}$ & $\begin{array}{c}18.3 \\
(32.4)\end{array}$ \\
\hline Poly(MA-alt-S-co-BMA)-2/organo-MMT & 115.0 & 15.6 & $\begin{array}{c}301 \\
(217)\end{array}$ & $\begin{array}{c}382 \\
(359)\end{array}$ & $\begin{array}{c}0.0 \\
(3.7)\end{array}$ & $\begin{array}{l}5.2 \\
(9.1\end{array}$ & $\begin{array}{c}25.6 \\
(38.4)\end{array}$ \\
\hline Poly(MA-alt-S-co-BMA)-3/organo-MMT & 90.1 & 26.2 & $\begin{array}{c}241 \\
(237)\end{array}$ & $\begin{array}{c}391 \\
(373)\end{array}$ & $\begin{array}{c}0.3 \\
(1.4)\end{array}$ & $\begin{array}{c}4.6 \\
(6.8)\end{array}$ & $\begin{array}{c}21.3 \\
(34.1)\end{array}$ \\
\hline Poly(IA-alt-S-co-BMA)-1/organo-MMT & 157.5 & 0.56 & 233 & 351 & 1.2 & 3.3 & 7.8 \\
\hline Pristine organo-MMT & 160.0 & 1.3 & 238 & 361 & 3.7 & 9.4 & 38.2 \\
\hline
\end{tabular}

aThis value corresponds to $T_{g}$

bThese values are related to the pure (co)terpolymers with different contents of BMA units (unit contents as in Table 1) 

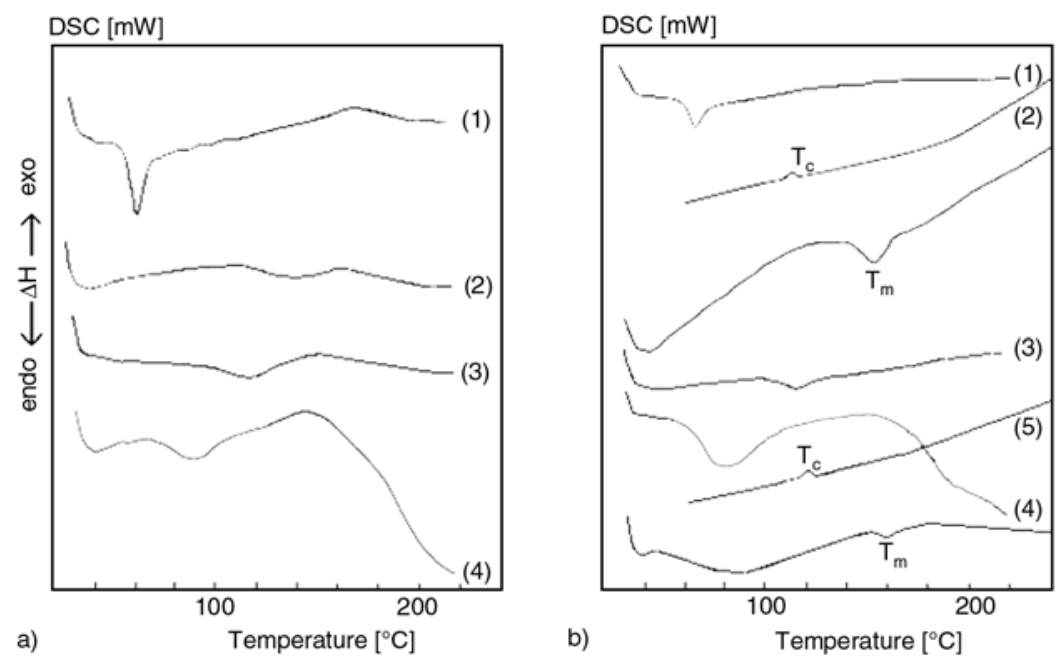

Figure 6. DSC curves of (a): (1) copolymer, (2) terpolymer-1, (3) terpolymer-2, and (4) terpolymer-3; (b): (1) copolymer/DMDA-MMT, (2) terpolymer-1/DMDA-MMT, (3) terpolymer-2/DMDA-MMT, (4) terpolymer-3/DMDAMMT, and (5) pristine DMDA-MMT

$200^{\circ} \mathrm{C}$ to room temperature in the DSC conditions. These results allow us to imply that these peaks are associated with glass transition or pseudo-melting temperature due to the side-chain $n$-butylester linkages which are able to form liquid crystalline structure. The terpolymers with different contents of BMA units exhibit typical $T_{g}$ behaviour, the values of which essentially decrease with increasing flexible $n$-butylester units in the terpolymers. Unlike C1-NC, terpolymer nanocomposites (T-1-NC, T-2-NC and T-3-NC) containing different mol\% of BMA units exhibit $T_{m}$ behaviour with characteristic recrystallization temperatures $\left(T_{c}\right)$ (Figure 6). The $\mathrm{Tm}$ values of these nanocomposites significantly depend on the BMA content in terpolymers. As seen from DSC curves (Figure 6) and data in Table 2, the $T_{m}$ values reduce from 140.1 to $94.8^{\circ} \mathrm{C}$ for virgin terpolymers and from 168.0 to $78.5^{\circ} \mathrm{C}$ for terpolymer/DMDA-MMT nanocomposites as a function of the content of flexible BMA linkages in terpolymers which is increased from 38.5 to $71.1 \mathrm{~mol} \%$. An increase of flexible hydrophobic $n$ butylester side-chain groups in terpolymer result with visible changes of enthalpy values $(\Delta H)$ of melting phase transition of nanocomposites. Poly(MA-co-BMA) and its nanocomposite exhibit typical glass-transition $\left(T_{g}\right)$ behaviour with $\Delta H$ (copolymer) $>\Delta H$ (copolymer/DMDA-MMT).

As seen from Table 2, terpolymer/nanocomposite (T-1-IA-NC) prepared by interlamellar terpolymerization of IA, S and BMA exhibit relatively higher thermal behaviour than poly(MA-alt-S-BMA)/
DMDA-MMT (T-1-NC) although the synthesized nanocomposites have an approximately similar composition and BMA unit content. This observed significant difference can be explained by the above mentioned structural factor, i.e., the formation of interlayer strong $\mathrm{H}$-bonding in the presence of IA monomer and its terpolymer chain.

Nanocomposites and virgin organo-MMT showed a similar melting and re-crystallization behaviour while $\Delta H$ melting increased (area of peak decreased) in the case of the formation of nanostructural sections. Unlike virgin (co)terpolymers, the nanocomposites exhibit crystallization behaviour and similar dependence on the content of BMA linkage in terpolymers. The observed broad endo-peak around $65-110^{\circ} \mathrm{C}$ for virgin DMDAMMT can be associated with the elimination of water trace. The weak endo- and exo-peaks observed in DSC curve of DMDA-MMT (Figure 6, curve b-3) can be related to the melting and crystallization processes of alkyl ammonium surfactant on the layered silicate surface, intensity ( $\Delta H$ value) of which significantly increases after interlamellar copolymerization due to the formation of interlayer complexes between functional copolymer and alkyl amine.

TGA-DTG analysis (Figure 7) indicated the multistep degradation of virgin co(ter)polymers, while their DMDA-MMT nanocomposites showed a single step degradation. This observed effect can be explained by the formation of a more compact and organized structure in synthesized co(ter)copoly- 

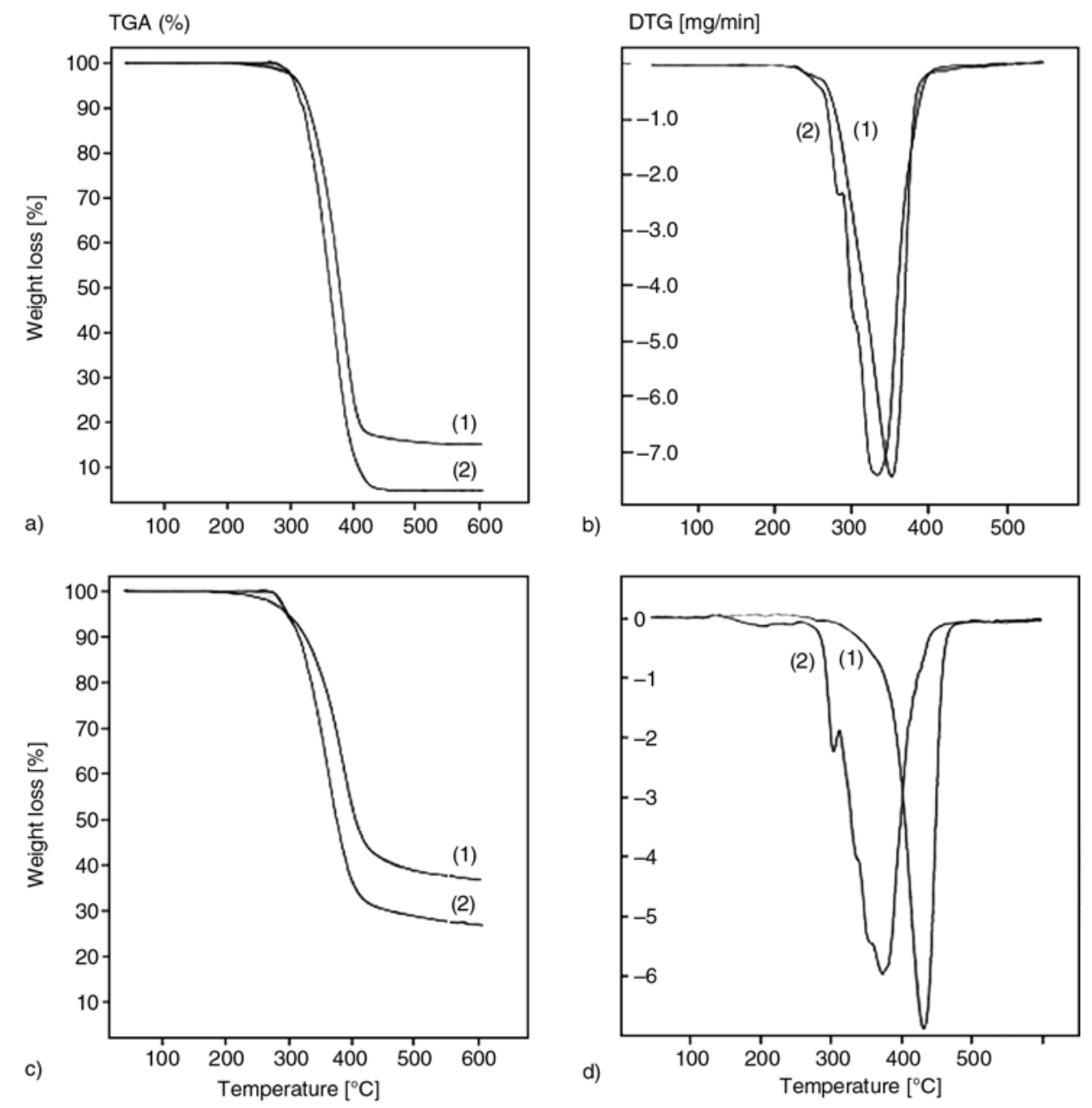

Figure 7. TGA (a and c) and DTG (b and d) curves of (1) (co)terpolymers and their (2) nanocomposites: (a)-1 and (b)-1 copolymer, (a)-2 and (b)-2 copolymer/DMDA-MMT, (c)-1 and (d)-1 terpolymer-1,(c)-2 and (d)-2 terpolymer1/DMDA-MMT

mer/organo-MMT hybrids. Obtained results related to the thermal behaviour of virgin (co)terpolymers and (co)terpolymers/DMDA-MMT hybrids prepared by complex-radical copolymerization and interlamellar copolymerization, respectively, are summarized in Table 2 . The comparative analysis of these results indicate that synthesized nanocomposites have relatively high $T_{m}$ and thermal stability (degradation temperature and weight loss), and exhibit both the melting phase transition and crystallization behaviour. The improvement in the degradation temperature was significantly due to the homogeneous dispersion of the silicate galleries in the functional copolymer matrix.

Comparative DSC and TGA analyses of prepared (co)terpolymers and their nanocomposites showed that (1) the terpolymers exhibit relatively higher values of $T_{g}$ and thermal stability as compared with copolymer due to the presence of the styrene units in the terpolymers and their high molecular weights (intrinsic viscosity values presented in Table 1), (2) unlike the copolymer composites, the terpolymer nanocomposites exhibit melting transition and crystallization behaviour, as well as higher thermal stability due to the formation of more compact structures, (3) terpolymer nanocomposite containing IA unit shows higher thermal behaviour as compared with MA containing nanocomposite due to the effect of strong $\mathrm{H}$-bonding (Figure 3 ) in the interlamellar terpolymerization, and (4) the change in the content of BMA flexible linkages in terpolymer/DMDA-MMT nanocomposites significantly influence the thermal behaviour of the nanocomposites, and can be described as a function for the correlation of these important properties through in situ self-plasticization in the formation of nanostructure with given thermal parameters. 


\subsection{Dynamic mechanical behaviour as a function of nanostructure formation}

Dynamic mechanical analysis (DMA) method allows us to determine the loss $\tan \delta$, the storage modulus $\left(G^{\prime}\right)$ and the loss modulus $\left(G^{\prime \prime}\right)$ as a function of temperature (Figure 8). The loss $\tan \delta$ is equal to the ratio of $G^{\prime \prime} / G^{\prime}$, which exhibits a higher response to the chemical and physical structural changes and phase transitions of polymer system; the storage modulus $G^{\prime}$ is associated with the elastic modulus of the polymer or its composite; the loss modulus $G^{\prime \prime}$ is related to the energy loss (damping factor) as a result of the friction of polymer chain movement. It is known that the decrease of $G^{\prime}$ is less dependent on the temperature changes, and this process proceeds very slowly as a function of temperature in the polymer system due to increased polymer chain mobility and flexibility [46]. As seen from DMA curves, this process con-
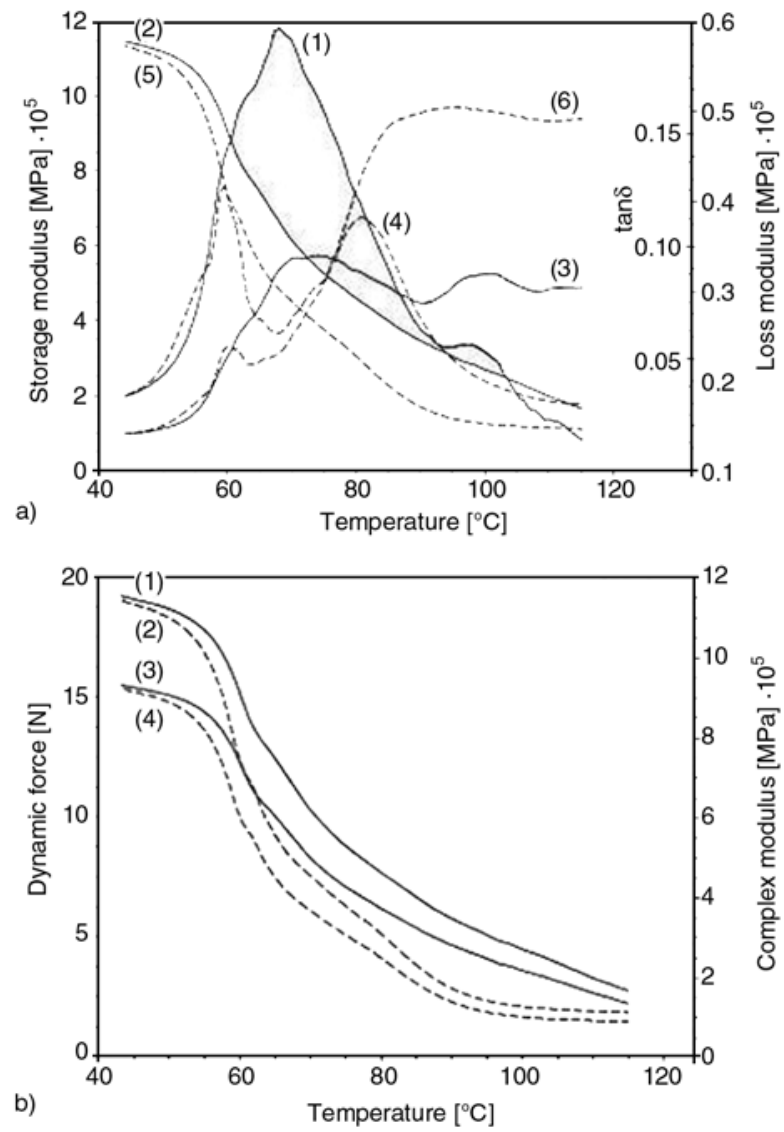

Figure 8. DMA curves of copolymer and its nanocomposite: (a) plots of (1 and 4) loss modulus $\left(G^{\prime \prime}\right),(2$ and 5) storage modulus $\left(G^{\prime}\right)$ and (3 and 6) $\tan \delta$ $\left(G^{\prime \prime} / G^{\prime}\right)$, and (b) (1 and 2) complex modulus and (3 and 4) dynamic force vs. temperature for poly(MA-co-BMA) (---) and poly(MA-coBMA)/DMDA-MMT nanocomposite (-) tinues until the glass transition region is approached, and then, $G^{\prime}$ decreases rapidly. In this isothermal condition, both the loss modulus $G^{\prime \prime}$ and loss $\tan \delta$ increase and go via a maximum. The glass transition temperature $\left(T_{g}\right)$ is determined as the temperature at which a maximum of loss $\tan \delta$ is observed. This is well known and is a more exact method for determination of $\mathrm{T}_{\mathrm{g}}$ values of polymers and their composites [47]. The glass transition region of polymeric materials can be broadened and shifted through various chemical and physical means [48].

Masenelli-Varlot et al. [49] proposed an interpretation of the DMA spectra, according which the intensity of the interfacial adhesion strength directly influences the intensity of the reinforcement above the $T_{g}$. This principle can also be used to characterize the microstructure of functional (co)terpolymer/DMDA-MMT nanocomposite in terms of interfacial adhesion strength. Comparative DMA analysis of pure poly(MA-co-BMA) and poly(MA-co-BMA)/DMDA-MMT nanocomposites (Figure 8a) indicates the following changes of DMA curves when copolymer is intercalated between organo-silicate layers by interlamellar radical copolymerization: (1) increase of the elastic modulus by more than $7.1 \%$, i.e., increase of the relaxation temperature from 58.3 up to $62.8^{\circ} \mathrm{C}$, (2) increase of the glass transition behavior from 60.1 to $74.2^{\circ} \mathrm{C}$, (3) unlike the pure copolymer, which has two peaks for the flexible BMA segments at $59.3^{\circ} \mathrm{C}$ and rigid MA units at $80.9^{\circ} \mathrm{C}$, respectively, copolymer nanocomposite exhibits a single broad peak of higher intensity at $67.9^{\circ} \mathrm{C}$; the significant increase in intensity of this peak indicates that relaxations of the flexible BMA segments decrease due to the increase of interfacial adhesion strength, i.e., the highest interaction occurs between anhydride units and organo-MMT. Plots of dynamic force (DF) and complex modulus (CM) vs. temperature are illustrated in Figure $8 \mathrm{~b}$. Observed significant difference $(\Delta)$ between these parameters $[\Delta=\mathrm{DF}$ (nanocomposite)-DF(copolymer) $]$ or $[\Delta=\mathrm{CM}$ (nanocomposite)-CM(copolymer)] above $T_{g}$, related to the copolymer and its nanocomposite, can be described as a function of the formation of nano-structural architecture in poly(MA-co-BMA)/DMDA-MMT system. It can be proposed that $\Delta$ value is also associated with interfacial adhesion strength depending on the flex- 
ibility and hydrophobic/hydrophilic balance of the functional polymer chains and their ability to form interfacial complexes. The maximum values of $\Delta$ were observed around $85-95^{\circ} \mathrm{C}$ corresponding to the lower values of DF and CM parameters.

\subsection{Nanostructure-XRD parameters relationship}

The basal spacing $\left(d_{001}\right)$ of copolymer, organoMMT and nanocomposites were determined from XRD measurements. XRD patterns of studied systems are illustrated in Figures 9 and 10, and the obtained XRD parameters are summarized in Table 3. It is well known that organo-silicates exhibit an effective nucleation behaviour in the

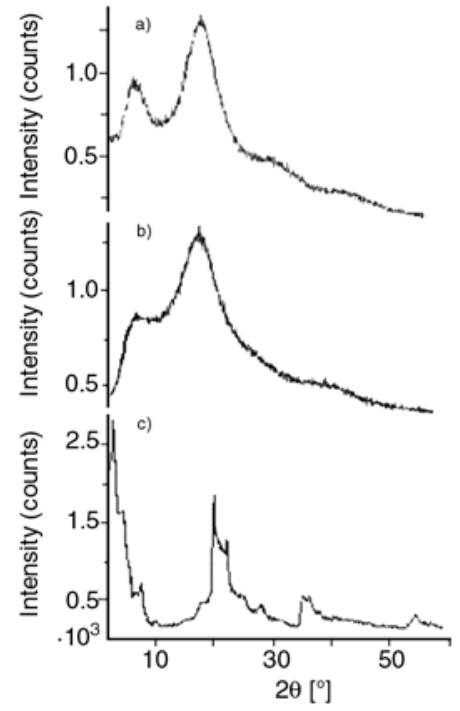

crystallization process and morphology formation of polymer nanocomposites [50, 51]; nanofillers can also change the crystallinity degree and crystallization rate, as well as provide the formation of thermodynamically stable crystalline phase in the polymer matrix $[52,53]$. As seen from Figure 9 , (co)terpolymers exhibit predominantly amorphous structure while their nanocomposites show characteristic semi-crystalline structure. IA-containing terpolymer nanocomposites (T-IA-NC) exhibit relatively higher crystallinity equal to $51 \%$ (Table 3 ). The position of the diffraction peaks, related to virgin amorphous (co)terpolymers, is not changed in the spectra of the (co)terpolymer/DMDA-MMT nanocomposites (Figure 10, Table 3). XRD patterns for the virgin DMDA-MMT clearly showed

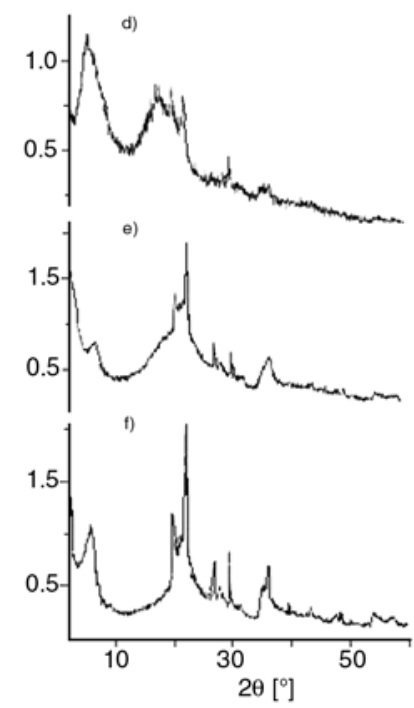

Figure 9. XRD patterns of (a) copolymer, (b) terpolymer-2, (c) pristine DMDA-MMT, (d) copolymer/DMDA-MMT, (e) terpolymer-2/DMDA-MMT, (f) terpolymer-IA/DMDA-MMT

Table 3. XRD parameters and crystallinity of the copolymer (C), terpolymers (T) and their nanocomposites (NCs)

\begin{tabular}{|c|c|c|c|c|c|c|c|c|}
\hline \multirow{2}{*}{$\begin{array}{c}\mathrm{C}(\mathrm{T}) \\
\text { and NCs }\end{array}$} & \multirow{2}{*}{$\begin{array}{l}2 \theta \\
{\left[{ }^{\circ}\right]}\end{array}$} & \multirow{2}{*}{$\begin{array}{c}\text { d-spacing } \\
\text { [̊]] }\end{array}$} & \multirow{2}{*}{$\begin{array}{l}\Delta \mathbf{d} \\
{[\AA]}\end{array}$} & \multirow{2}{*}{$\begin{array}{c}\text { Intensity } \\
\text { (counts) }\end{array}$} & \multirow{2}{*}{$\begin{array}{l}\text { ED }^{\mathbf{a}} \\
{[\%]}\end{array}$} & \multicolumn{2}{|c|}{ Peak area (S) } & \multirow{2}{*}{$\chi_{\mathrm{c}}[\%]$} \\
\hline & & & & & & $\mathbf{S}_{\mathrm{a}}$ & $\mathbf{S}_{\mathrm{c}}$ & \\
\hline $\mathrm{C}-1$ & 6.74 & 13.10 & 0 & 1349 & - & 26208 & 4866 & 15.6 \\
\hline $\mathrm{C}-1-\mathrm{NC}$ & 2.22 & 35.68 & 17.73 & 1613 & 52.4 & 11250 & 2760 & 19.7 \\
\hline $\mathrm{T}-1$ & 6.53 & 13.52 & 0 & 1271 & - & 20642 & 2137 & 9.4 \\
\hline T-1-NC & 2.31 & 30.87 & 12.92 & 1688 & 53.4 & 16123 & 5092 & 24.0 \\
\hline $\mathrm{T}-2$ & 6.92 & 12.76 & 0 & 1497 & - & 30517 & 4625 & 13.2 \\
\hline T-2-NC & 2.58 & 34.22 & 16.27 & 1750 & 54.3 & 16606 & 6173 & 27.1 \\
\hline $\mathrm{T}-3$ & 8.19 & 10.79 & 0 & 1310 & - & 32817 & 3737 & 10.2 \\
\hline T-3-NC & 2.10 & 33.20 & 15.25 & 2069 & 58.4 & 9279 & 4447 & 32.4 \\
\hline T-IA-NC & 2.04 & 44.14 & 26.19 & 2100 & 100 & 6712 & 6986 & 51.0 \\
\hline $\begin{array}{l}\text { Pristine } \\
\text { organo-MMT }\end{array}$ & $\begin{array}{l}2.78 \\
4.92 \\
7.38\end{array}$ & $\begin{array}{l}31.75 \\
17.95 \\
11.97\end{array}$ & $\begin{array}{l}- \\
- \\
-\end{array}$ & $\begin{array}{c}2769 \\
1475 \\
750^{b}\end{array}$ & - & 7217 & 10047 & 58.2 \\
\hline
\end{tabular}

${ }^{a} \mathrm{ED}(\%$ exfoliation degree) values were calculated using the Equation (4)

'This peak disappears in the spectra of all the nanocomposites due to complete exfoliation 


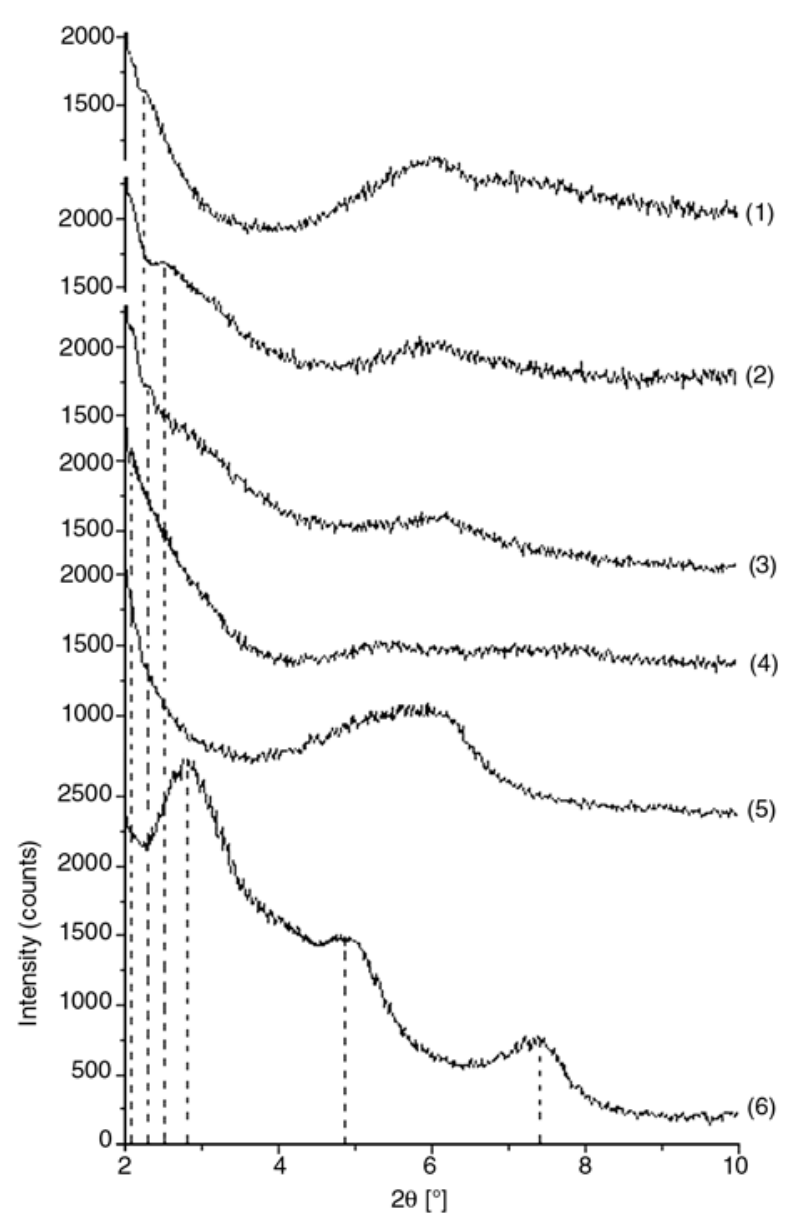

Figure 10. XRD patterns of nanocomposites:

(1) poly(MA-co-BMA)/DMDA-MMT,

(2) poly(MA-alt-S-co-BMA)-1/DMDA-MMT,

(3) poly(MA-alt-S-co-BMA)-2/DMDA-MMT,

(4) poly(MA-alt-S-co-BMA)-3/DMDA-MMT,

(5) poly(IA-alt-S-co-BMA)-1/DMDA-MMT and (6) pristine DMDA-MMT

two broad diffraction peaks at $2.78^{\circ}(d$-spacing $31.75 \AA$ ) and $4.92^{\circ}$ ( $d$-spacing $\left.17.95 \AA\right)$ due to the $d_{001}$ basal reflections, which disappear or significantly shift to lower $2 \theta$ regions in nanocomposites. The weak peak for the basal spacing $\left(d_{001}\right)$ at $7.38^{\circ}$ (11.97 $\AA$ ) in DMDA-MMT pattern can be related to non-modified silicate plates. Usually this peak for pure MMT appears at $7.02 \theta$ ( $d$-spacing $12.4 \AA$ ) [54]. Figure 10 shows the XRD patterns of organoMMT itself and (co)terpolymer/DMDA-MMT nanocomposites containing $5 \mathrm{wt} \%$ of organoMMT. The intense peak at $2 \theta=2.78^{\circ}(d=31.75 \AA)$, relatively broad peak $2 \theta=4.92^{\circ}(d=17.95 \AA)$ and peak with low intensity at $2 \theta=7.38^{\circ}(d=10.97 \AA)$ in the pattern of DMDA-MMT, are associated with basal spacing for modified and small amount of non-modified silicate layers $\left(2 \theta=7.38^{\circ}\right)$, respectively. According to Mermut [55] and Önal [56], $d_{(001)}$ values scattered around $12-14,14-18 \AA$ and $>18 \AA$ are due to the monolayer, bilayer and pseudotrilayer alkyl ammonium configurations, respectively, in the 2:1 layers of organo-MMTs including Viscobent SB-1 [56]. In the case of nanocomposites (patterns 2-4), the original organoMMT peaks at $2 \theta=7.38^{\circ}$ disappear, and peaks at 2.78 and $4.92^{\circ}$ shift to lower $2 \theta$ regions (for the coand terpolymer nanocomposites, patterns 2,4 and 6). As seen from Figure 10 and Table 3, these observed changes clearly indicate the significant increase of interlamellar distance $\left(\Delta d=d_{n c}-d_{0}>\right.$ $15 \AA$ ) due to in situ processing through complex formation or $\mathrm{H}$-bonding between anhydride/acid groups and surface alklammonium cations. This can also produce a driving force necessary for the effective intercalation/exfoliation. The interlamellar complex-formation and (co)terpolymerizaton reactions easily proceed onto alkyl amine modified silicate layers, after ending of which can also proceed onto non-modified silicate layers. In the latter case, anhydride (or carboxylic group which occurs by the hydrolysis of the MA unit) and acid (IA) monomers and their (co)terpolymers can also form interlamellar complexes with the non-modified silicate layers containing oxygen atoms and hydroxyl groups through strong H-bonding [8].

It was observed that the exfoliation degree (ED) depends on the type of alkyl amine configuration, i.e., on the degree of intensity change of basal $d$-spacing peaks. Taking into consideration the three basal peak intensities for the organo-MMT before $\left(I_{0}\right)$ and after interlamellar (co)terpolymerizations $\left(I_{e}\right)$, the values of intercalation/exfoliation degree $(\mathrm{ED} \%)$ were calculated, and obtained results were presented in Table 3. The peak with weak intensity at $7.38^{\circ}(d$-spacing $11.97 \AA$ for nonmodified 2:1 layers) disappears in the XRD spectra for all the nanocomposites (Figure 8) due to the complete exfoliation process. This observed fact confirms the above mentioned proposed mechanism. It was also observed that the crystallinity of the (co)terpolymers/DMDA-MMT nanocomposites depends on the degree of exfoliation. Relatively high crystalline copolymer-NC and terpolymer-IA-NC exhibit higher values of ED (Table 3). A visible increase in ED for terpolymer-MA-NC nanocomposites increases the crystallinity of these NCs. This phenomenon may be explained by the effect of complex formation between functional 
polymer chains and organo-silicate layers on the local chain folding and crystallization process in the (co)terpolymer matrix. The ED values visibly increase with increasing content of the flexible BMA linkages in terpolymer-MA-NCs, in spite of the decreasing content of the complex-forming anhydride/acid units. This fact can be explained by self-plasticization effect of side-chain $n$-butylester groups in terpolymers that increases the dispersion of the organo-MMT into matrix.

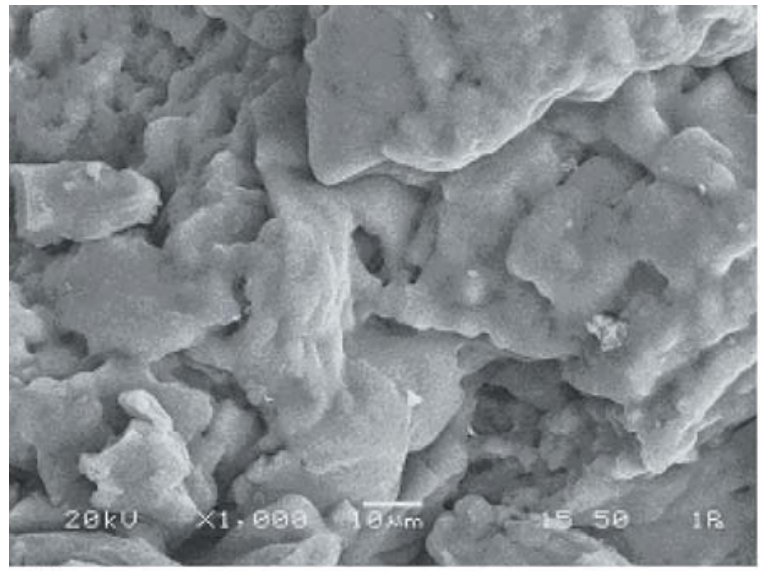

a)

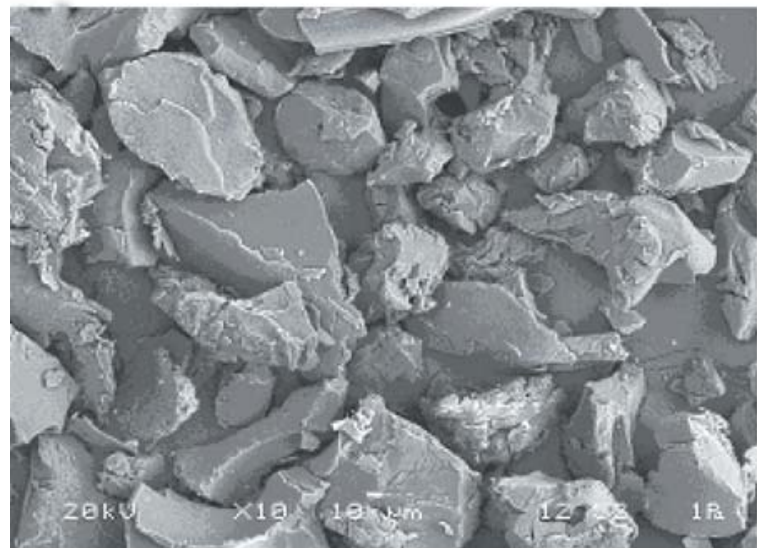

c)

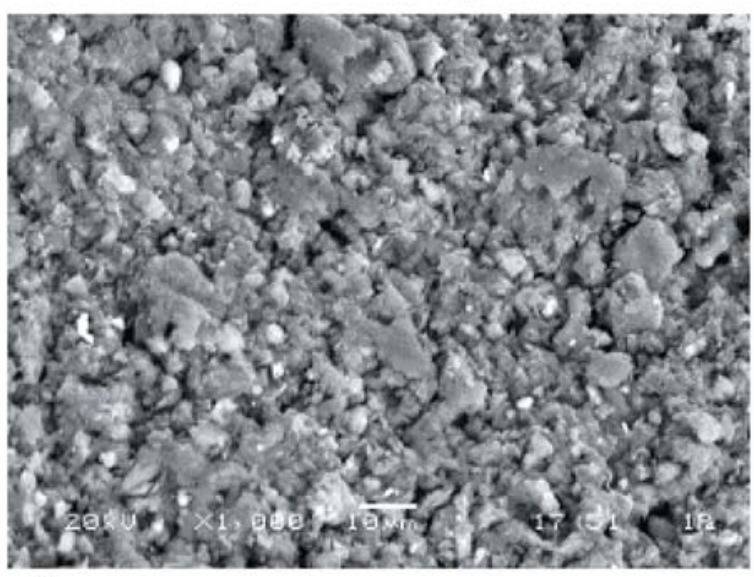

Figure 11. SEM patterns of (a) terpolymer-2, (b) terpolymer-2/DMDA-MMT, (c) terpolymer-3, (d) terpolymer-3/ DMDA-MMT and (e) terpolymer-IA/DMDA-MMT

\subsection{Surface morphology of nanocomposites}

Figure 11 shows the SEM image of the terpolymers containing different amounts of BMA units and their nanocomposites prepared by interlamellar copolymerization. SEM images of this hybrid composites inferred that the size of the particles significantly depends on the content of flexible BMA linkages and type of interfacial interaction. The increase of BMA content in terpolymers provides the facile exfoliation of polymer chains into

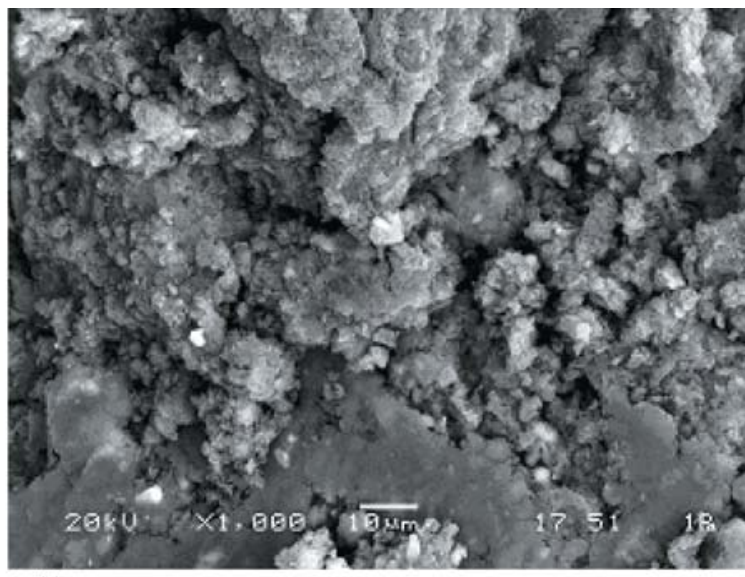

b)

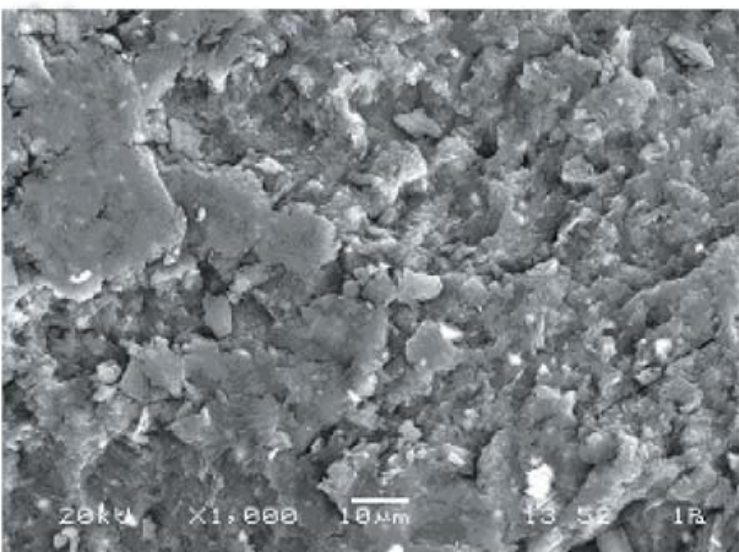

d) 
DMDA-MMT galleries and formation of relatively high dispersed particles. In the case of poly(IA-coS-BMA)/DMDA-MMT nanocomposite (e), the formation of well dispersed particles was observed due to the strong H-bonding between free carboxylic acid of IA monomer or terpolymer chain and alkyl amine on the silicate layers.

\section{Conclusions}

This work has attempted to develop novel polymer layered silicate nanocomposites by complex-radical interlamellar (co)terpolymerization of intercalated MA...DMDA-MMT and IA...DMDA-MMT monomer complexes with $\mathrm{S}$ and BMA. It was shown that the intercalation/exfoliation behaviour of functional (co)terpolymers, and therefore, the formation of nano-structure in the studied systems depend on the hydrophilic/hydrophobic balance in amphiphilic terpolymers, amount of flexible BMA linkages and interlayer complex-formation between anhydride/acid units and surface alkylammonium fragments (Figure 1). The comparative analysis of structure-composition-property relationship of the virgin (co)terpolymers and their nanocomposites showed significant increase in the thermal and dynamic mechanical behaviour and crystallinity of synthesized nanocomposites due to the homogeneous dispersion of the silicate nano-galleries in the functional (co)terpolymer matrix. Unlike amorphous virgin (co)terpolymers the nanocomposites exhibit crystallization behaviour and considerably improved thermal properties depending on the structure (presence of anhydride or acid moieties) and composition of terpolymers, especially on the content of flexible side-chain $n$-butylester linkages. The significant increase in $d$-spacing (intergallery distance) and degree of exfoliation indicates the formation of nano-structure in the studied (co)terpolymer/DMDA-MMT systems. Obtained results show that the formation of nanoarchitectures in the studied (co)terpolymer/DMDA-MMT systems predominantly proceeds through interlamellar complex-formation and in situ intercalation/exfoliation processing.

Synthesized polymer hybrids may be also used as reactive compatibilizer-nanofillers for the thermoplastic polymer blends, especially for the acrylic and styrene polymer-based systems, and to prepare the various nanomaterials in melt by reactive extru- sion in situ processing. This will be a subject of our future investigations.

\section{Acknowledgements}

This study was carried out in according to the Polymer Science and Engineering Program of the Chemical Engineering Department, Hacettepe University (HU). The support of the TÜB?TAK (Turkish National Scientific and Technical Research Council) through TBAG-HD/249-107T238 project is gratefully acknowledged.

\section{References}

[1] Alexandre M., Dubois P.: Polymer-layered silicate nanocomposites: Preparation, properties and uses of a new class of materials. Material Science and Engineering: Reports, 28, 1-63 (2000).

[2] Sinha Ray S., Okamoto M.: Polymer/layered silicate nanocomposites: A review from preparation to processing. Progress in Polymer Science, 28, 1539-1641 (2003).

[3] Moniruzzaman M., Winey K. I.: Polymer nanocomposites containing carbon nanotubes. Macromolecules, 39, 5194-5205 (2006).

[4] Rzaev Z. M. O., Yilmazbayhan A., Alper E.: A onestep preparation of compatibilized polypropylenenanocomposites by reactive extrusion processing. Advances in Polymer Technology, 26, 41-55 (2007).

[5] Utracki L. A., Sepehr M., Boccaleri E.: Synthesic, layered nanoparticles for nanocomposites (PNCs). Polymers for Advanced Technology, 18, 1-37 (2007).

[6] Krawczak P.: Compounding and processing of polymer nanocomposites: From scientific challenges to industrial stakes. Express Polymer Letters, 1, 188-188 (2007).

[7] Da Z. L., Zhang Q. Q., Wu D. M., Yang D. Y., Qiu F. $\mathrm{X}$.: Synthesis, characterization and thermal properties of inorganic-organic hybrid. Express Polym Letters, 1, 698-703 (2007).

[8] Kawasumi M., Hasegawa N., Kato M., Usuki A., Okada A.: Preparation and mechanical properties of polypropylene-clay hybrids. Macromolecules, 30, 6333-6338 (1997).

[9] Okada A., Usuki A.: The chemistry of polymer-clay hybrids. Material Science and Engineering, part C: Biomimetic Materials, Sensors and Systems, 3, 109 115 (1995).

[10] Messersmith P. B., Giannelis E. P.: Synthesis and barrier properties of poly( $\varepsilon$-caprolactone)-layered silicate nanocomposites. Journal of Polymer Science, Part A: Polymer Chemistry, 33, 1047-1057 (1995).

[11] Burnside S. D., Gianellis E. P.: Synthesis and properties of new poly(dimethylsiloxane) nanocomposites. Chemistry of Materials, 7, 1597-1600 (1995). 
[12] Liu Y-L., Wu C-S., Chiu Y-S., Ho W-H.: Preparation, thermal properties, and flame retardance of epoxy-silica hybrid resins. Journal of Polymer Science, Part A: Polymer Chemistry, 41, 2354-2367 (2003).

[13] Tang Y., Hu Y., Wang S. F., Gui Z., Chen Z., Fan W. C.: Preparation and flammability of ethylene-vinyl acetate copolymer/montmorillonite nanocomposites. Polymer Degradation and Stabilization, 78, 555-559 (2002).

[14] Yeh J-M., Liou S-J., Lai C-Y., Wu P-C.: Enhancement of corrosion protection effect in polyaniline via the formation of polyaniline-clay nanocomposite materials. Chemistry of Materials, 13, 1131-1136 (2001).

[15] Yeh J-M., Liou S-J., Lin C-Y., Cheng C-Y., Chang YW., Lee K-R.: Anticorrosively enhanced PMMA-clay nanocomposite materials with quaternary alkylphosphonium salt as an intercalating agent. Chemistry of Materials, 14, 154-161 (2002).

[16] Doh J. G., Cho I.: Synthesis and properties of polystryrene-organoammonim montmorillonite hybrid. Polymer Bulletin, 41, 511-518 (1998).

[17] Zeng Q. H., Wang D. Z., Lu G. Q.: Synthesis of polymer-montmorillonite nanocomposites by in situ intercalative polymerization. Nanotechnology, 13, 549553 (2002).

[18] Liu G., Zhang L., Zhao D., Qu X.: Bulk polymerization of styrene in the presence of organomodified montmorillonite. Journal of Applied Polymer Science, 96, 1146-1152 (2005).

[19] Biswas M., Sinha Ray S.: Preparation and evaluation of composites from montmorillonite and some heterocyclic polymers. 1 . Poly $(N$-vinylcarbazole)-montmorillonite nanocomposite system. Polymer, 39, 64236428 (1998).

[20] Friedlander H. Z.: Spontaneous polymerization in and on clay. ACS Division Polymer Chemistry Reprints, 4, 300-306 (1963).

[21] Di J., Sogah D. Y.: Intergallery living polymerization using silicate-anchored photoiniferter. A versatile preparatory method for exfoliated silicate nanocomposites. Macromolecules, 39, 1020-1028 (2006).

[22] Stadtmueller L. M., Ratinac K. R., Ringer S. P.: The effect of intragallery polymerization on the structure of PMMA-clay nanocomposites. Polymer, 46, 95749584 (2005).

[23] Li H-M., Chen H-B.: Synthesis and characterization of poly $(N$-n-butylmaleimide)-clay nanocomposites. Material Letters, 57, 3000-3004 (2003).

[24] Yan-wu Z., Jun-qing J.: Preparation of low-molecularweight polyamide 6 /hydrotalcite intercalated nanocomposites via in situ polymerization. Express Polymer Letters, 1, 98-103 (2007).

[25] Vaysse C., Guerlou-Demourgues L., Delmas C., Duguet E.: Tentative mechanisms for acrylate intercalation and in situ polymerization in nickel-based layered double hydroxides. Macromolecules, 37, 45-51 (2004).
[26] Vaysse C., Guerlou-Demourgues L., Duguet E., Delmas C.: Acrylate intercalation and in situ polymerization in iron-, cobalt-, or manganese-substituted nickel hydroxides. Inorganic Chemistry, 42, 4559-4567 (2003).

[27] Vieille L., Moujahid E. M., Taviot-Gueho C., Cellier J., Besse J-P., Leroux F.: In situ polymerization of interleaved monomers: A comparative study between hydrotalcite and hydrocalumite host structures. Journal Physical Chemistry of Solids, 65, 385-393 (2004).

[28] Cao C., Huang F., Cao C. T., Li J., Zhu H.: Synthesis of carbon nitride nanotubes via a catalytic-assembly solvothermal route. Chemistry of Materials, 16, 52135215 (2004)

[29] Yu F., Yao K., Shi L., Wan W., Zhong Q., Fu Y., You $\mathrm{X}$.: Investigation of acrylic acid polymerization in novel two-dimensional space with regular amino groups of layered aminipropylsilica. Chemistry of Materials, 19, 3412-3418 (2007).

[30] Xu M. Z., Coi Y. S., Kim Y. K., Wang K. H., Chung I. J.: Synthesis and characterization of exfoliated poly(styrene-co-methyl methacrylate)/clay nanocomposites via emulsion polymerization with AMPS. Polymer, 44, 6387-6395 (2003).

[31] Noh M. H., Jang L. W., Lee D. C.: Intercalation of styrene-acrylonitrile copolymer in layered silicate by emulsion polymerization. Journal of Applied Polymer Science, 74, 179-188 (1999).

[32] Liu G., Zhang L., Gao C., Qu X.: Copolymerization of styrene with $N$-phenyl maleimide in the presence of montmorillonite. Journal of Applied Polymer Science, 98, 1932-1937 (2005).

[33] Liu J.-H., Zhang J., Li F.: Flame retardancy of styrene and butyl acrylate/montmorillonite nanocomposites. Polymer Material Science and Engineering, 21, 156159 (2005).

[34] Al-Esaimi M. M.: Reaction catalyzed by montmorillonite: Polymerization of methyl methacrylate. Journal of Applied Polymer Science, 64, 367-372 (1997).

[35] Liu G-D., Li X., Zhang L-C., Qu X-G., Liu P., Yang L., Gao C-H.: Thermal analysis of solution copolymers of styrene with $N$-phenylmaleimide. Journal of Applied Polymer Science, 83, 417-422 (2002).

[36] Wang D., Zhu J., Yao Q., Wilkie C. A.: A comparison of various methods for the preparation of polystyrene and poly(methyl methacrylate) clay nanocomposites. Chemistry of Materials, 14, 3837-3843 (2002).

[37] Wang D., Zhu J., Yao Q., Wilkie C. A.: In-situ reactive blending to prepare polystyrene-clay and polypropylene-clay nanocomposites. Polymer Degradation and Stability, 80, 171-182 (2003).

[38] Rzaev Z. M. O., Guner A., Can H. K., Assici A.: Reaction of some anhydride-containing copolymers with $\gamma$ aminopropyltriethoxysilane. Polymer, 42, 5599-606 (2001). 
[39] Can H. K., Rzaev Z. M. O., Güner A.: Synthesis and characterization of new hydrogels on the basis of water-soluble maleic anhydride copolymers with $\gamma$ aminopropyltriethoxysilane. Journal of Applied Polymer Science, 90, 4009-4015 (2003).

[40] Rzayev Z. M. O.: Nanotechnology methods in polymer engineering. in " $4^{\text {th }}$ Nanoscience and Nanotechnology Conference Proceedings, Ankara, Turkey’ 154 (2007).

[41] Rabek J. F.: Experimental methods in polymer chemistry. Wiley, New York (1980).

[42] Figueruelo J. E., García-Lopera R., Monzó I. S., Abad C., Campos A.: Determination of $\chi$ from liquid-liquid phase data in ternary polymer systems (solvent/polymer/polymer) with hydrogen bonding. Express Polymer Letters, 2, 313-329 (2008).

[43] Cowie J. M. G.: Alternating copolymers. Plenum, New York (1985).

[44] Hendricks S. B.: Base exchange of the clay mineral montmorillonite for organic cations and its dependence upon adsorption due to van der Waals forces. Journal of Physical Chemistry, 45, 65-81 (1941).

[45] Sun S. F.: Physical chemistry of macromolecules. John Wiley and Sons, New Jersey (2004).

[46] Gong X., Liu J., Baskaran S., Voise R. D., Young J. S.: Surfactant-assisted processing of carbon nanotube/ polymer composites. Chemistry of Materials, 12, 1049-1052 (2000).

[47] Mark H. F., Bikales N. M., Overberger C. C., Menges G.: Encyclopedia of polymer science and engineering. John Wiley and Sons, New York (1987).
[48] Sperling L. H.: Introduction to physical polymer science. John Wiley and Sons, New York (1992).

[49] Masenelli-Varlot K., Vigier G., Vermogen A., Gauthier C., Cavaille J. Y.: Quantitative structural characterization of polymer-clay-nanocomposites and discussion of an 'ideal' microstructure, leading to the highest mechanical reinforcement. Journal of Polymer Science, Part B: Polymer Physics, 45, 1243-1351 (2007).

[50] Fornes T. D., Paul D. R.: Crystallization behavior of nylon 6 nanocomposites. Polymer, 44, 3945-3961 (2003).

[51] Harrats C., Groeninckx G.: Features, questions and future challenges in layered silicates clay nanocomposites with semicrystalline polymer matrice. Macromolecular Rapid Communication, 29, 14-26 (2008).

[52] Lewin N. H., Pearce M. E., Levon K., Mey-Maron A., Zammarano M., Wilkie C. A., Jang B. N.: Nanocomposites at elevated temperatures: Migration and structural changes. Polymers for Advanced Technology, 17, 226-234 (2006).

[53] Ajayan M., Schadler L. S., Braun P. V.: Nanocomposite science and technology. Wiley-VCH, Weinheim (2003).

[54] Cho J. M., Paul D. R.: Nylon 6 nanocomposites by melt compounding. Polymer, 42, 1083-1094 (2001).

[55] Mermut A. R.: Layer charge characteristics of 2:1 silicate clay minerals. The Clay Mineral Society, Aurora (1994).

[56] Önal M.: Examination of some commercial sorptive organobentonites. Turkish Journal of Chemistry, 31, 579-588 (2007). 